\title{
Application of Fully Decoupled Parity Equation in Fault Detection and Identification of DC Motors
}

\author{
C. W. Chan, Song Hua, and Zhang Hong-Yue, Senior Member, IEEE
}

\begin{abstract}
A multiple fault detection and identification method based on fully decoupled parity equations for dynamic systems with known linear and unknown nonlinear terms is presented. The fully decoupled parity equation vectors is derived and it is shown that the residuals generated from it are decoupled from other faults and the unknown nonlinear term and are sensitive only to specific actuator or sensor faults. The condition for the existence of the equation is also given. From the residuals generated from the fully decoupled parity equation, the faults are estimated using the recursive least-squares method. The performance of the proposed method is illustrated by applying it to detect, isolate, and identify faults in a simulated de motor.
\end{abstract}

Index Terms-Fault detection and identification, parity equation, recursive least-squares method.

\section{INTRODUCTION}

$\mathbf{F}$ AULT detection, isolation, and identification are important to improve the safety and reliability of practical control systems. A number of techniques for fault diagnosis can be found in well-known books in this research area [1]-[3]. A general approach to detect faults is based on residuals generated from the parity equation [4]-[9]. The residuals are small, if the system is operating normally, but are large, if there are faults in the system. If the residuals are sensitive to specific faults, then these faults can be isolated and identified. The design of the parity equation for systems with modeling errors arising from changes in the operating point is presented in [8]. An optimal robust parity equation for generating residuals maximally sensitive to faults and minimally sensitive to modeling errors is derived in [9]. This approach is further refined in [10] and a criterion is constructed for determining a set of parity vectors with different robustness properties in fault detection. The optimal parity vector for isolating faults is proposed in [11] and [12]. However, the identification of the faults has not been considered.

The unknown input observer (UIO) is another popular fault diagnosis approach for systems with an unknown nonlinearity.

Manuscript received August 16, 2004; revised March 30, 2005. Abstract published on the Internet May 18, 2006. This work was supported in part by the China Natural Science Foundation under Grant 60234010 and Grant 60572185, and in part by the Hong Kong Special Administrative Region Research Grant Council Grant HKU 7050/02E.

C. W. Chan is with the Department of Mechanical Engineering, The University of Hong Kong, Hong Kong (e-mail: mechan@hkucc.hku.hk).

S. Hua and Z. Hong-Yue are with the School of Automation Science and Electrical Engineering, Beihang University, Beijing 100083, China (e-mail: songhua7421@sohu.com; buaa_301_216@sina.com).

Digital Object Identifier 10.1109/TIE.2006.878304
In [13], the uncertainty is estimated and the estimate is used to discount the effect of the nonlinear uncertainty. Observers are proposed in [14] and [15] to decouple the effect of the unknown inputs, and are then used in fault diagnosis schemes [16], [17]. Although fault diagnosis based on the UIO can handle nonlinear systems, few results are available in the literature to use this approach to identify faults. However, it is important to identify faults, especially for fault accommodation.

To compute the residuals from the general parity equation, it is necessary to obtain first an accurate mathematic model of the system. For this reason, it has mainly been applied to linear systems, where accurate models are more readily available. However, as accurate models for nonlinear systems are more difficult to obtain in practice, and as the parity equation is sensitive to noise and model uncertainties, the parity equation derived using existing approaches cannot be readily applied to nonlinear and uncertainty systems. In this paper, a new approach is presented not only to detect and isolate faults, but also to identify faults for systems with know linear and unknown nonlinear terms. In practical control systems, there are two common faults: the actuator fault and the sensor fault [18]. In this paper, a fully decoupled parity equation is derived and the residuals generated from it are decoupled from the system state, the unknown nonlinearity, and other faults, but only sensitive to specific sensor or actuator faults. For an actuator, the common faults are either it fails completely, or it is jammed [19]. Sensor faults are more complicated. Bias and/or drifting can occur, as well as precision degradation or complete failure [19]. It is shown in [19] and [20] that these faults can be described by a linear fault model, and faults can be identified from the parameters of this model. As the fault model is linear, its parameters can be identified from the residuals using the recursive least-squares method. To illustrate the performance of the proposed method, it is applied to a simulated de motor, and it is shown that the proposed method is able to detect, isolate, and identify the faults.

The paper is organized as follows. In Section II, the conditions for the residuals generated from the fully decoupled parity equations to be decoupled from the system state, the unknown nonlinearity and other faults, but only sensitive to specific sensor or actuator faults are derived. The isolation of actuator or sensor fault based on the fully coupled parity equation is presented in Section III, followed by the identification of actuator or sensor faults based on the linear fault model in Section IV. The implementation and the performance of the proposed method is demonstrated by a simulated dc motor with a shunt field circuit, and it is shown that the actuator or sensor faults can be successfully isolated and identified. 


\section{Fully Decoupled Parity Equation}

Practical dynamic systems can often be represented by a known linear state-space model with unknown nonlinearity. The known linear part can be obtained from the physical construction of the system, while the unknown nonlinear part includes the uncertainty and the unknown inputs such as modeling errors and external disturbances. The nonlinear discrete dynamic system can be described by [11]

$$
\left\{\begin{array}{l}
x(k+1)=A x(k)+B u(k)+F w(x, u, k)+\varsigma(k) \\
y(k)=C x(k)+G \psi(x, u, k)+\xi(k)
\end{array}\right.
$$

where $x(k) \in R^{n}$ is the state, $u(k) \in R^{p}$ is the input, $y(k) \in$ $R^{q}$ is the sensor output, $w(x, u, k) \in R^{r}$ and $\psi(x, u, k) \in$ $R^{r 1}$ are the unknown nonlinear part of the system, $\varsigma(k)$ and $\xi(k)$ are noise with Gaussian distribution, and $A, B, C$, $F$, and $G$ are known matrices with appropriate dimensions. Let $w(k)=w(x, u, k)$ and $\psi(k)=\psi(x, u, k)$. For $s>0$, the second equation in (1), or the measurement equation is given by [11]

$$
Y(k)=H_{0} x(k-s)+H_{c} U(k)+H_{w} W(k)+H_{\psi} \Psi(k)
$$

where $Y(k)$ is the normal sensor output, $s$ is the order of the parity space, $Y(k)=\left[y^{\mathrm{T}}(k-s) \ldots y^{\mathrm{T}}(k)\right]^{\mathrm{T}}, U(k)=$ $\left[u^{\mathrm{T}}(k-s) \ldots u^{\mathrm{T}}(k)\right]^{\mathrm{T}}, \quad W(k)=\left[w^{\mathrm{T}}(k-s) \ldots w^{\mathrm{T}}(k)\right]^{\mathrm{T}}$, $\Psi(k)=\left[\psi^{\mathrm{T}}(k-s) \ldots \psi^{\mathrm{T}}(k)\right]^{\mathrm{T}}$, and $H_{0}, H_{c}, H_{w}$, and $H_{\psi}$ are

$$
\begin{aligned}
& H_{0}=\left[\begin{array}{c}
C \\
C A \\
\vdots \\
C A^{s-1} \\
C A^{s}
\end{array}\right] \\
& H_{c}=\left[\begin{array}{cccccc}
0 & & & & \cdots & 0 \\
C B & 0 & & & \cdots & 0 \\
C A B & C B & 0 & & \cdots & 0 \\
\vdots & & & & & \\
C A^{s-2} B & \cdots & \cdots & C B & 0 & 0 \\
C A^{s-1} B & C A^{s-2} B & \cdots & C A B & C B & 0
\end{array}\right] \\
& H_{w}=\left[\begin{array}{cccccc}
0 & & & & \cdots & 0 \\
C F & 0 & & & \cdots & 0 \\
C A F & C F & 0 & & \cdots & 0 \\
\vdots & & & & & \\
C A^{s-2} F & \cdots & \cdots & C F & 0 & 0 \\
C A^{s-1} F & C A^{s-2} F & \cdots & C A F & C F & 0
\end{array}\right] \\
& H_{\psi}=\left[\begin{array}{c}
G \\
G \\
\vdots \\
G
\end{array}\right] \text {. }
\end{aligned}
$$

Definition 1: The parity space $V$ is defined as follows [12], [20]

$$
V=\left\{v \mid v^{\mathrm{T}} H_{0}=0\right\}
$$

where $v$ is the parity vector with order $s$, and $H_{0}$ and $s$ are defined in (3).
Definition 2: The parity equation at time $k$ is given by

$$
r(k)=v^{\mathrm{T}}\left[Z(k)-H_{c} U_{c}(k)\right]
$$

where $r(k)$ is the residual, $U_{c}(k)=\left[u_{c}(k-s) \ldots u_{c}(k)\right]^{\mathrm{T}}$ is the normal input to the actuator, and $Z(k)=[Z(k-$ $s) \ldots Z(k)]^{\mathrm{T}}$ is the sensor output that may contain faults.

From (5), any actuator or sensor fault is included in $r(k)$. For a normal actuator, $U_{c}(k)=U(k)$, and for a normal sensor, $Z(k)=Y(k)$. To isolate and identify faults, the residuals generated by (5) should be sensitive only to the specific fault.

Definition 3: If parity vector $v$ satisfies

$$
V^{*}=\left\{v \mid v^{\mathrm{T}} H^{*}=0\right\}
$$

where $H^{*}$ is to decouple $v$ from the state, unknown nonlinear term and other faults. Then, $V^{*}$ is the fully decoupled parity space and $v$ is the fully decoupled parity vector.

The conditions under which the fully decoupled parity (5) can be used to detect, isolate, and identify faults are given below.

Lemma 1: Faults can be detected, isolated, and identified by $r(k)$ given by the fully decoupled parity (5), if there exists a fully decoupled parity vector $v$, such that $r(k)$ is:

1) decoupled from the states of systems;

2) decoupled from the unknown nonlinear parts $W(k)$ and $\Psi(k)$, which may contain the unknown inputs and the uncertainty;

3) sensitive only to special actuator faults or sensor faults, but decoupled from other faults.

Proof: Condition (1) can be obtained readily from (4). For a system operating normally, $r(k)$ obtained from (2) is

$$
\begin{aligned}
r(k)= & v^{\mathrm{T}}\left[Y(k)-H_{c} U_{c}(k)\right] \\
= & v^{\mathrm{T}}\left[H_{0} x(k-s)+H_{c} U(k)+H_{w} W(k)\right. \\
& \left.\quad+H_{\Psi} \Psi(k)-H_{c} U_{c}(k)\right] \\
= & v^{\mathrm{T}}\left[H_{0} x(k-s)+H_{w} W(k)+H_{\Psi} \Psi(k)\right] .
\end{aligned}
$$

From (4), $r(k)$ is decoupled from the system state when the system is normal. If the unknown nonlinear terms $W(k)$ and $\Psi(k)$ are identically zero, $r(k)$ obtained from (7) is zero only if the system is normal. If $v^{\mathrm{T}}\left[H_{w} W(k)+H_{\psi} \Psi(k)\right]$ is identically zero, then condition (2) follows, and hence $r(k)$ is decoupled from $W(k)$ and $\Psi(k)$. If conditions (1) and (2) are satisfied, $r(k)$ is nonzero only if the system is faulty. Therefore, faults can be detected from $r(k)$, as follows

$$
\|r(k)\|= \begin{cases}\geq r_{h} & \text { faulty } \\ <r_{h} & \text { normal }\end{cases}
$$

where $r_{h}$ is the threshold obtained from experience based on the safety and reliability requirement for the system. However, the residual $r(k)$ generated from (5) satisfies only conditions (1) and (2), and can only be used to detect fault, but not to isolate it. This is because $r(k)$ is a function of all actuator faults or sensor faults. However, if conditions (3) is satisfied, then $r(k)$ will only be sensitive to specific faults, and insensitive to any other faults. Consequently, $r(k)$ can also be used to isolate and identify 
faults using a fault model, as described later. Conditions (2) and (3) are the key to derive the fully decoupled parity equation. The diagnosis of multiple actuator and sensor faults based on Lemma 1 is presented below.

\section{ISOLATION OF FAULTS}

To isolate a fault, the residual must be sensitive only to that fault, but insensitive to any other faults. The design of $H^{*}$ to ensure the fully decoupled vector $v$ satisfies condition (3) for an actuator fault or a sensor fault is presented in this section.

\section{A. Isolation of Actuator Faults}

To isolate the actuator fault with the assumption that the sensors are operating normally, the parity vector must satisfy (4) to ensure the residuals are decoupled from the system state. The residuals are decoupled from the unknown nonlinear terms $W(k)$ and $\Psi(k)$, if [21]

$$
v^{\mathrm{T}} H_{w}=0 \quad v^{\mathrm{T}} H_{\psi}=0 .
$$

For actuator $i$, the parity vector must satisfy the following equation:

$$
v_{i}^{\mathrm{T}} H_{c i}=0, \quad i=1,2, \ldots, p
$$

where $p$ is the number of actuators in the dynamic system, $\nu_{i}$ is the parity vector sensitive to the $i$ th actuator, and $H_{c i}$ is

$$
H_{c i}=\left[\begin{array}{cccccc}
0 & 0 & & \cdots & & 0 \\
C B^{*} & 0 & 0 & & & \vdots \\
C A B^{*} & C B^{*} & 0 & 0 & & \\
\vdots & & & & & \\
C A^{s-2} B^{*} & \cdots & \cdots & C B^{*} & 0 & 0 \\
C A^{s-1} B^{*} & C A^{s-2} B^{*} & \cdots & C A B^{*} & C B^{*} & 0
\end{array}\right]
$$

where $B^{*}$ is the matrix after removing the $i$ th column from $B$, which corresponds to the $i$ th actuator. It follows that $H^{*}$ is given by

$$
H^{*}=\left[\begin{array}{llll}
H_{0} & H_{w} & H_{\psi} & H_{c i}
\end{array}\right] .
$$

From (6), (9), and (10), the fully decoupled parity vector sensitive to the $i$ th actuator must satisfy

$$
v_{i}^{\mathrm{T}}\left[\begin{array}{llll}
H_{0} & H_{w} & H_{\psi} & H_{c i}
\end{array}\right]=0 .
$$

The parity equation constructed by the parity vector satisfying (13) is referred to as the fully decoupled parity equation sensitive to a specific actuator. The condition for the existence of nonzero solutions of (13) is given in Theorem 1.

Theorem 1: Let the column rank of $\left[H_{0} H_{w} H_{\psi} H_{c i}\right]$ be $n_{x}$. The necessary and sufficient condition for (13) to have nonzero solutions is

$$
s>\frac{n_{x}}{q}-1
$$

and the sufficient condition is

$$
s>\frac{n+r 1}{q+1-(r+p)}-1, \quad q>(r+p)-1
$$

where $n$ is the dimension of the system states, $q$ and $p$ are, respectively, the number of sensors and actuators, and $r$ and $r 1$ are, respectively, the dimension of the unknown nonlinear term in (1).

Proof: The left null space and hence the nonzero solution of (13) exists, if and only if the number of rows of $\left[\begin{array}{llll}H_{0} & H_{w} & H_{\psi} & H_{c i}\end{array}\right]$ is greater than $n_{x}$. Since the dimension of $H_{0}, H_{w}, H_{\psi}$, and $H_{c i}$ are, respectively, $(s+1) q \times n$, $(s+1) q \times(s+1) r 1,(s+1) q \times r 1$, and $(s+1) q \times(s+1)$. $(p-1)$, hence the dimension of $\left[\begin{array}{llll}H_{0} & H_{w} & H_{\psi} & H_{c i}\end{array}\right]$ is given by $(s+1) q \times[n+r 1+(s+1)(r+p-1)]$. Therefore, the necessary and sufficient condition for the existence of nonzero solution is: $(s+1) q>n_{x}$, i.e., $s>\left(n_{x} / q\right)-1$.

Further, if the number of rows of $\left[H_{0} H_{w} H_{\psi} H_{c i}\right]$ is greater than the number of columns, then the nonzero solution of (13) is certain to exist, giving the sufficient condition: $(s+1) q>$ $n+r 1+(s+1)(r+p-1)$.

From Theorem 1, the order $s$ can be selected first before constructing the matrices $H_{0}, H_{w}, H_{\psi}$, and $H_{c i}$. From (13), if $q>(r+p)-1$, an appropriate data window $s$ can be selected to obtain the fully decoupled parity vector for constructing $H_{0}, H_{w}, H_{\psi}$, and $H_{c i}$. Clearly, if $q>n+r 1+(r+p)$, then $s=0$.

\section{B. Isolation of Sensor Faults}

Similarly, it is assumed that the actuators are operating normally when detecting sensor faults. The fully decoupled parity equation is constructed such that the residuals generated are sensitive only to specific sensor faults, but insensitive to other sensor faults. For sensors from 1 to $q$, the fully decoupled parity equation given by (5) is obtained for each sensor using $H_{0 i}, H_{c i}, H_{w i}$, and $H_{\psi i}$ and with $C$ and $G$ replaced by $C_{i}$ and $G_{i}$, where $C_{i}$ and $G_{i}$, are obtained from $C$ and $G$ by setting the $i$ th row to 0 , which corresponds to the $i$ th sensor.

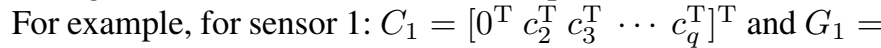
$\left[\begin{array}{lllll}0^{\mathrm{T}} & g_{2}^{\mathrm{T}} & g_{3}^{\mathrm{T}} & \cdots & g_{q}^{\mathrm{T}}\end{array}\right]^{\mathrm{T}}$, where $c_{j}$ and $g_{j}(j=1,2, \ldots, q)$ are the $j$ th row vector of matrices $C$ and $G$. If the sensors are operating normally, then

$$
r_{i}(k)=v_{i}^{\mathrm{T}}\left[H_{0 i} x(k-s)+H_{w i} W(k)+H_{\psi i} \Psi(k)\right] .
$$

From (16), for $r_{i}(k)$ to be decoupled from the system state, $v_{i}$ must satisfy

$$
v_{i}^{\mathrm{T}} H_{0 i}=0 .
$$

The parity vector $v_{i}$ should also satisfy the following conditions if it is decoupled from $W(k)$ and $\Psi(k)$ :

$$
v_{i}^{\mathrm{T}} H_{w i}=0 \quad v_{i}^{\mathrm{T}} H_{\psi i}=0
$$

and $H^{*}$ becomes

$$
H^{*}=\left[\begin{array}{lll}
H_{0} & H_{w i} & H_{\psi i}
\end{array}\right] .
$$


It follows that if the parity vector is insensitive to the $i$ th sensor, then

$$
v_{i}^{\mathrm{T}}\left[H_{0 i} \quad H_{w i} \quad H_{\psi i}\right]=0 .
$$

For $v_{i}$ to be insensitive to the $i$ th sensor, the elements corresponding to the $i$ th sensor in $H_{0 i}, H_{c i}, H_{w i}$, and $H_{\psi i}$ must be 0 . The parity equation given by (20), referred to as the fully decoupled parity equation, is insensitive to the $i$ th sensor, if the element of $v_{i}$ corresponding to the $i$ th sensor is 0 . The condition for the existence of $v_{i}$ is given below.

Theorem 2: Let $n_{i}$ be the number of the independent columns in $\left[H_{0 i}^{*} H_{w i}^{*} H_{\psi i}^{*}\right]$. The necessary and sufficient condition for the existence of nonzero solution of (20) is

$$
s>\frac{n_{i}}{q-1}-1
$$

and the sufficient condition is

$$
s>\frac{n+r 1}{q-1-r}-1, \quad q>r+1
$$

where $H_{0 i}^{*}, H_{w i}^{*}$, and $H_{\psi i}^{*}$ are obtained from $H_{0 i}, H_{w i}$, and $H_{\psi i}$ by removing the $i$ th row of $C_{i}, D_{i}$, and $G_{i}$, which is a " 0 " vector.

The proof of this theorem is similar to that of Theorem 1. From (21) and (22), $s$ is selected, and $H_{0 i}, H_{c i}, H_{w i}$, and $H_{\psi i}$ are then constructed.

\section{IDENFICATION OF FAULTS}

It is shown in [18] and [20] that the actuator and/or sensor faults, such as varying scaling factor and constant bias, can be described by

$$
z=\eta y+\lambda
$$

where $z$ is measurement with a fault, $y$ the output without fault, $\eta$ the scaling factor, and $\lambda$ the bias. If an actuator or a sensor is operating normally, then $\eta=1$ and $\lambda=0$. For constant output fault, $\eta=0$, and $\lambda$ is a nonzero constant. For a scaling factor fault, $\eta \neq 1$ and $\lambda=0$, and for a constant bias fault, $\eta=1$ and $\lambda \neq 1$. The estimation of the parameters $\eta$ and $\lambda$ from residuals generated from the fully decoupled parity equation will be discussed in this section.

\section{A. Identification of Actuator Faults}

If the $i$ th actuator is faulty, then (23) becomes

$$
u_{i}(k)=\eta_{i}(k) u_{i c}(k)+\lambda_{i}(k)
$$

where $u_{i}(k)$ is the input of the $i$ th actuator, $u_{i c}(k)$ is the normal input, $\eta_{i}(k)$ is the scaling factor, and $\lambda_{i}(k)$ is the bias. From the residuals generated from the fully decoupled parity equations, the parameters of the fault model (24) can be estimated by using the recursive least-squares method. From (2) and (5), the residuals that are sensitive to the $i$ th actuator are given by

$$
\begin{aligned}
r_{i}(k)=v_{i}^{\mathrm{T}}\left[\left(H_{0} x(k-s)+H_{c} U(k)\right.\right. & \\
& \left.\left.+H_{w} W(k)+H_{\psi} \Psi(k)\right)-H_{c} U_{c}(k)\right] .
\end{aligned}
$$

From $\quad(11), \quad \nu_{i}^{\mathrm{T}} H_{0} x(k-s)=0, \quad \nu_{i}^{\mathrm{T}} H_{w} W(k)=0 \quad$ and $\nu_{i}^{\mathrm{T}} H_{\psi} W(k)=0$, then

$$
r_{i}(k)=v_{i}^{\mathrm{T}}\left[H_{c} U(k)-H_{c} U_{c}(k)\right] .
$$

Also, $v_{i}^{\mathrm{T}} H_{c i}=0$, where $H_{c i}$ is obtained by removing the $i$ th column of $B$ in $H_{c}$. Since $r_{i}(k)$ obtained from (26) is sensitive only to the $i$ th actuator and insensitive to the other actuators, changes in the input of other actuators do not affect $r_{i}(k)$. Therefore, it can be assumed that the scaling factor and the bias of the fault model of the other actuators are the same as that for the $i$ th actuator. As the fault model of actuator at $s+1$ is unchanged from the last data window at $s$, then (24) becomes

$$
U(k)=\eta_{i}(k) U_{c}(k)+\lambda_{i}(k) E
$$

where $E=\left[\begin{array}{llll}1 & 1 & \cdots & 1\end{array}\right]^{\mathrm{T}}$ is a vector with a dimension of $(s+1) p$. Rewriting (26) gives

$$
\begin{aligned}
r_{i}(k) & =v_{i}^{\mathrm{T}}\left[H_{c}\left(\eta_{i}(k) U_{c}(k)+\lambda_{i}(k) E\right)-H_{c} U_{c}(k)\right] \\
& =v_{i}^{\mathrm{T}}\left[\left(\eta_{i}(k)-1\right) H_{c} U_{c}(k)+H_{c} \lambda_{i}(k) E\right] \\
& =\phi_{i}(k) \theta_{i}(k) .
\end{aligned}
$$

Rewriting (28) to include modeling and measurement noise $n(k)$ gives

$$
r_{i}(k)=\phi_{i}(k) \theta_{i}(k)+n(k)
$$

where $\phi_{i}(k)=\left[\nu_{i}^{\mathrm{T}} H_{c} U_{c}(k) \nu_{i}^{\mathrm{T}} H_{c} E\right]$ and $\theta_{i}(k)=\left[\left(\eta_{i}(k)-\right.\right.$ 1) $\left.\lambda_{i}(k)\right]^{\mathrm{T}}$. Assuming $n(k)$ is a zero mean white noise with a covariance matrix of $R(k)$, then from (29), $\hat{\theta}(k)$ can be obtained by the recursive least-squares method [23]:

$$
\begin{aligned}
K(k) & =P(k-1) \phi^{\mathrm{T}}(k)\left[I+\phi(k) P(k-1) \phi^{\mathrm{T}}(k)\right]^{-1} \\
\hat{\theta}(k) & =\hat{\theta}(k-1)+K(k)[r(k)-\phi(k) \hat{\theta}(k-1)] \\
P(k) & =P(k-1)-K(k) \phi(k) P(k-1)
\end{aligned}
$$

where $K(k)$ and $P(k)$ are, respectively, the gain matrix and the error covariance matrix, and $I$ is the unit matrix. From (29), the fault of the $i$ th actuator appears in the residuals, if the fully decoupled parity vector also satisfies

$$
v_{i}^{\mathrm{T}} H_{c} \neq 0 .
$$

This result is summarized in the following theorem.

Theorem 3: The residual generated from the fully decoupled parity equation can detect, isolate, and identify the actuator faults, if the fully decoupled parity vector satisfies (13) and (33), i.e.,

$$
v_{i}^{\mathrm{T}}\left[\begin{array}{llll}
H_{0} & H_{w} & H_{\psi} & H_{c i}
\end{array}\right]=0 \quad \text { and } \quad v_{i}^{\mathrm{T}} H_{c} \neq 0 .
$$

\section{B. Identification of Sensor Faults}

The fault model of the $i$ th sensor can be described by [22]

$$
z_{i}(k)=y_{i}(k)+f_{i}(k)
$$


where $z_{i}(k)$ is the measured output of the $i$ th sensor and $y_{i}(k)$ is the output without fault. In matrix form, (34) becomes

$$
z(k)=y(k)+f(k)
$$

where

$$
\begin{aligned}
z(k) & =\left[\begin{array}{llll}
z_{1}(k) & z_{2}(k) & \cdots & z_{q}(k)
\end{array}\right]^{\mathrm{T}} \\
y(k) & =\left[\begin{array}{llll}
y_{1}(k) & y_{2}(k) & \cdots & y_{q}(k)
\end{array}\right]^{\mathrm{T}} \\
f(k) & =\left[\begin{array}{llll}
f_{1}(k) & f_{2}(k) & \cdots & f_{q}(k)
\end{array}\right]^{\mathrm{T}} .
\end{aligned}
$$

Assuming the fault model of sensor is unchanged from the last data window $s+1$, then

$$
Z(k)=Y(k)+I^{*} f(k)
$$

where $Z(k)=\left[z^{\mathrm{T}}(k-s), z^{\mathrm{T}}(k-s+1), \ldots, z^{\mathrm{T}}(k)\right]^{\mathrm{T}}$ is the actual sensor output, $I^{*}=\left[\begin{array}{llll}I_{0} & I_{0} & \cdots & I_{0}\end{array}\right]^{\mathrm{T}}$ is a matrix with dimension $(s+1) q \times q$ and $I_{0}$ is a $q \times q$ identity matrix. The residual insensitive to the $i$ th sensor is

$$
\begin{aligned}
r_{i}(k) & =v_{i}^{\mathrm{T}}\left[Z_{i}(k)-H_{c i} U(k)\right] \\
& =v_{i}^{\mathrm{T}}\left[Y_{i}(k)+I^{*} f^{i}(k)-H_{c i} U(k)\right]
\end{aligned}
$$

where $y_{i}$ and $f_{i}$ are replaced by 0 in $Y_{i}(k)$ and $f_{\mathrm{i}}(k)$. When the actuators are operating normally, the residual insensitive to the $i$ th sensor can be obtained from (16) and (37)

$$
\begin{array}{r}
r_{i}(k)=v_{i}^{\mathrm{T}}\left[H_{0 i} x(k-s)+H_{c i} U(k)+H_{w i} W(k)\right. \\
\left.+H_{\psi i} \Psi(k)+I^{*} f^{i}(k)-H_{c i} U(k)\right] .
\end{array}
$$

From (20)

$$
v_{i}^{\mathrm{T}} H_{0 i} x(k-s)=0, v_{i}^{\mathrm{T}} H_{w i} W(k)=0, v_{i}^{\mathrm{T}} H_{\psi i} W(k)=0
$$

hence

$$
\begin{aligned}
r_{i}(k) & =v_{i}^{\mathrm{T}} I^{*} f^{i}(k) \\
r(k) & =v I^{*} f(k) \\
& =\phi(k) \theta(k)
\end{aligned}
$$

where $r(k)=\left[\begin{array}{llll}r_{1}(k) & r_{2}(k) & \cdots & v_{q}\end{array}\right]^{\mathrm{T}}$ and $v=\left[\begin{array}{llll}v_{1} & v_{2} & \cdots & v_{q}\end{array}\right]^{\mathrm{T}}$. Rewriting (40) to include the measurement noise $n(k)$ yields

$$
r(k)=\phi(k) \theta(k)+n(k)
$$

where $\theta(k)=f(k), \phi(k)=v I^{*}$, and $n(k)$ is defined in (29). From (41), sensor faults can be estimated by the recursive leastsquare algorithms, as described previously.

\section{EXAMPLE}

Fault diagnosis of dc motors has attracted considerable interest, as they are often used in practical control systems [24], [25]. In this paper, the proposed approach is used to detect and identify faults in the dc motor. A dc motor with a shunt field

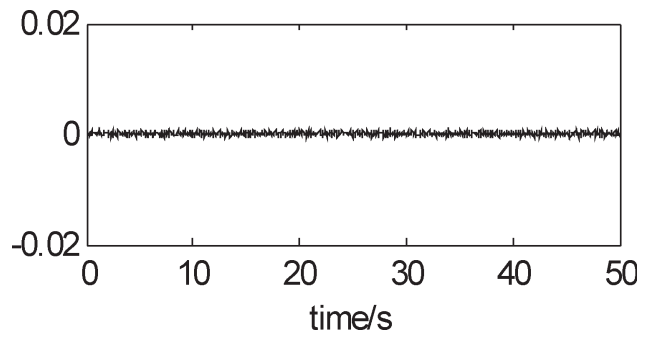

Fig. 1. Residual sensitive to actuator under normal operating conditions.

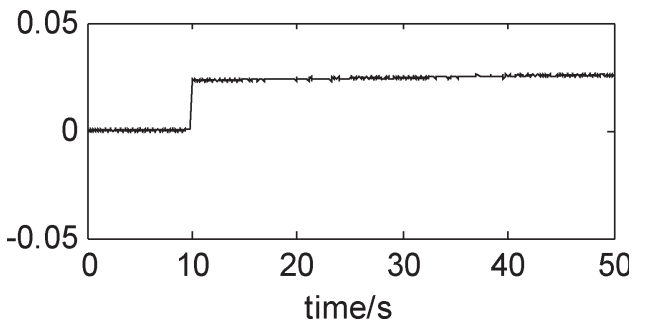

Fig. 2. Residual sensitive to actuator when fault occurred at $t=10 \mathrm{~s}$.

circuit can be described by the nonlinear ordinary differential equations [26]

$$
\begin{cases}\dot{i}_{f}=-\frac{R_{f}}{L_{f}} i_{f}+\frac{1}{L_{f}} V, & i_{f}(0)=i_{f 0} \\ \dot{i}_{a}=-\frac{R_{a}}{L_{a}} i_{a}-\frac{M}{L_{a}} i_{f} \omega_{r}+\frac{1}{L_{a}} V, & i_{a}(0)=i_{a 0} \\ \dot{\omega}_{r}=\frac{M}{J} i_{f} i_{a}-\frac{D}{J} \omega_{r}, & \omega_{r}(0)=\omega_{r 0}\end{cases}
$$

where $i_{f}$ is the field current, $i_{a}$ the armature current, $\omega_{r}$ the angular velocity, $V$ the input voltage, $R_{f}$ and $L_{f}$ the field resistance and inductance, $R_{a}$ and $L_{a}$ the armature resistance and inductance, $M$ the mutual inductance between $L_{f}$ and $L_{a}$, and $D$ and $J$ the viscous damping and the moment of inertia of the load. Let $x=\left[\begin{array}{ll}i_{f} & i_{a} \omega_{r}\end{array}\right]^{\mathrm{T}}$ be the state and $y=$

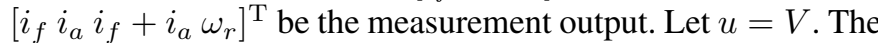
discrete state-space equation derived from (42) is [27]

$$
\begin{aligned}
x(k+1)= & {\left[\begin{array}{ccc}
0.8825 & 0 & 0 \\
0 & 0.6839 & 0 \\
0 & 0 & 0.9948
\end{array}\right] x(k) } \\
& +\left[\begin{array}{c}
0.002235 \\
0.0832 \\
0
\end{array}\right] u(k)+\left[\begin{array}{ll}
0 & 0 \\
1 & 0 \\
0 & 1
\end{array}\right] w(k)
\end{aligned}
$$

$$
y(k)=\left[\begin{array}{lll}
1 & 0 & 0 \\
0 & 1 & 0 \\
1 & 1 & 0 \\
0 & 0 & 1
\end{array}\right] x(k)
$$

where the unknown nonlinear term $w$ is defined as: $w(k)=$ $\left[\begin{array}{l}\varphi_{1}(k) \\ \varphi_{2}(k)\end{array}\right]$, and $\varphi_{1}(k)$ and $\varphi_{2}(k)$ are the discrete versions of $\left(-M / L_{a}\right) i_{f} \omega_{r}$ and $(M / J) i_{f} i_{a}$ in (42).

From (43) and (44), there are one actuator and four sensors. As $R(k)$ is assumed known, it is set to a $2 \times 2$ diagonal matrix with 0.02 along its diagonal in the simulation. The parity vectors can be obtained from (13) and (20), and residuals sensitive to a specific fault can be computed by (5). The fault models can be estimated from (29) and (41) by the recursive 

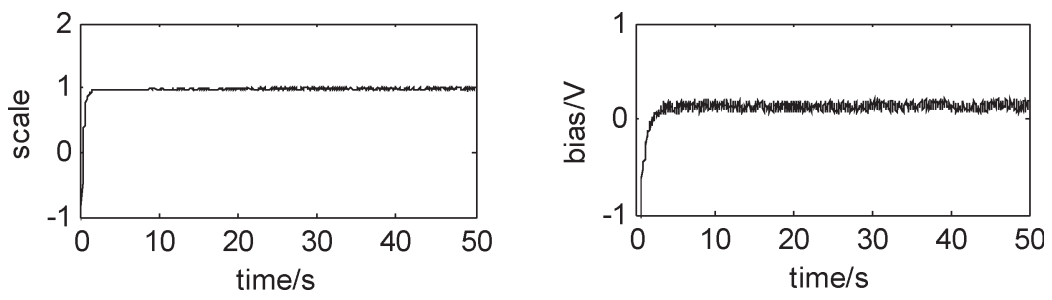

Fig. 3. Estimated parameters of fault model under normal operating conditions.
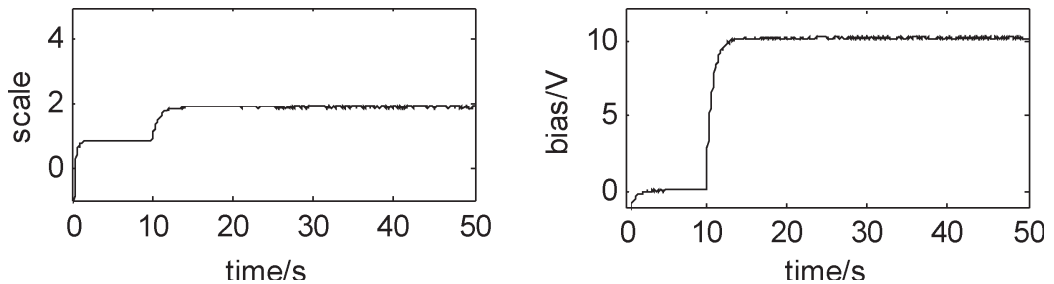

Fig. 4. Estimated parameters of the fault model when actuator fault occurred at $t=10 \mathrm{~s}$.
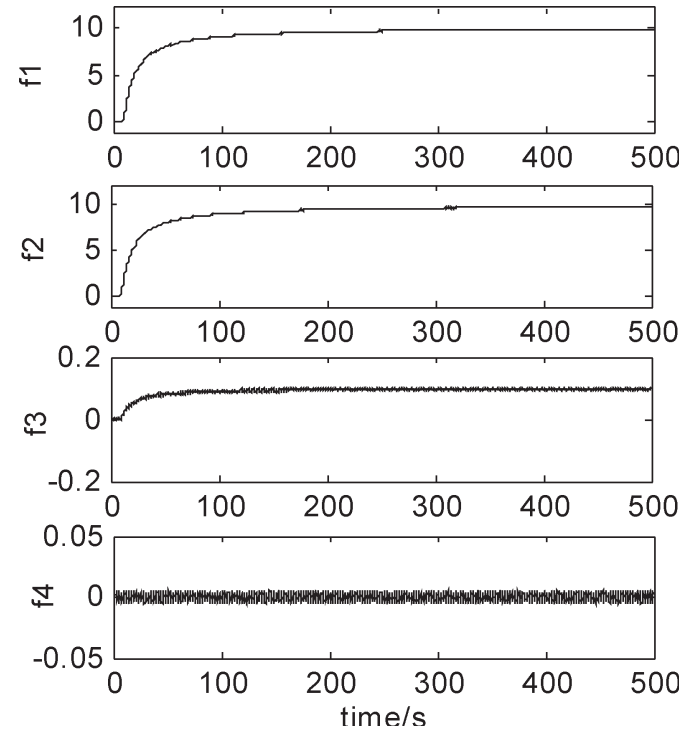

Fig. 5. Estimation of sensor faults when faulty occurred at $t=10 \mathrm{~s} f=$ $\left[\begin{array}{llll}10 & 10 & 0.1 & 0\end{array}\right]^{\mathrm{T}}$.

least-squares method. Since the constant bias fault is common in control systems [14], it will be used to validate the proposed technique in the simulation.

\section{A. Identification of Actuator Faults}

The matrix $H_{c}$ for the actuator is

$$
H_{c}=\left[\begin{array}{cccccccc}
0 & 0 & 0 & 0 & 0 & 0 & 0 & 0 \\
0 & 0 & 0 & 0 & 0.0022 & 0.0823 & 0.0845 & 0
\end{array}\right]^{\mathrm{T}} .
$$

The residuals sensitive to specific actuator fault before the fault occurs are shown in Fig. 1. When a fault given by the fault model (20) with $\eta=2$ and $\lambda=10$ occurred at $10 \mathrm{~s}$, the residuals are shown in Fig. 2. The identification of the fault under normal operating condition is shown in Fig. 3, and under faulty condition in Fig. 4. Clearly, the residuals generated from the fully decoupled parity equation is sensitive only to the specific actuator fault, and is independent of the system state
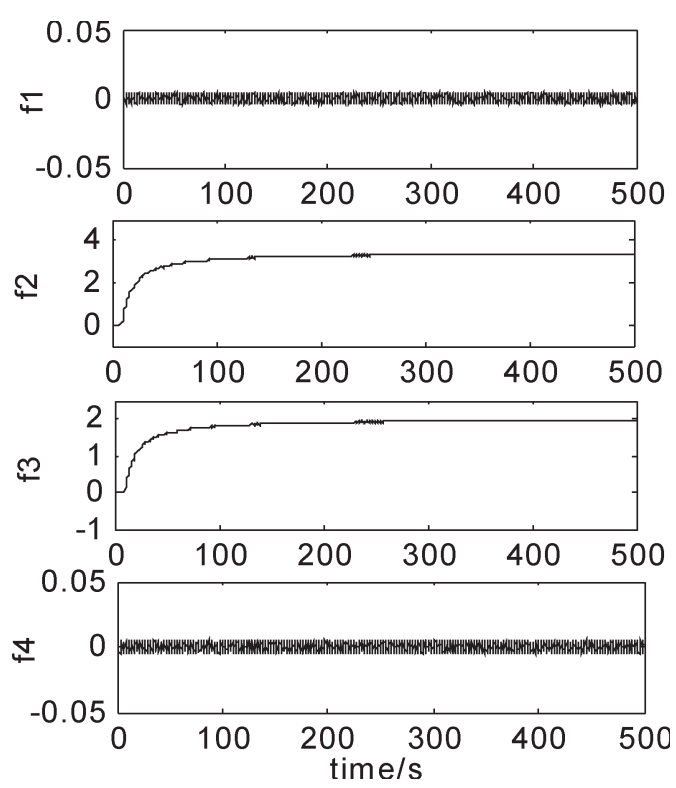

Fig. 6. Estimation of sensor faults when fault occurred at $t=10 \mathrm{~s}, f=$ $\left[\begin{array}{llllll}0 & 3.5 & 2 & 0\end{array}\right]^{\mathrm{T}}$.

and the unknown nonlinear term, indicating that the actuator fault can be detected, isolated, and identified.

\section{B. Identification of Sensor Faults}

The matrices $H_{c i}(i=1,2,3,4)$ for sensor 1 to sensor 4 are computed, as shown at the top of the next page.

Consider a step offset with a magnitude of 10 occurring in sensors 1 and 2, and 0.1 in sensors 3 and 4 after $10 \mathrm{~s}$. The estimated parameters of the fault model are shown in Fig. 5, and are close to the actual fault. Next, consider the case when sensors 1 and 4 are operating normally, while sensors 2 and 3 have developed a fault with a step offset of 3.5 and 2, respectively, after $10 \mathrm{~s}$. The estimated faults are shown in Fig. 6, showing that the estimated faults are close to the actual ones, illustrating that the proposed method is able to detect, isolate, and identify successfully sensor faults. 


$$
\begin{aligned}
H_{C 1} & =\left[\begin{array}{lllllccccccccccccccccc}
0 & 0 & 0 & 0 & 0 & 0.0823 & 0.0845 & 0 & 0 & 0.0563 & 0.0583 & 0 & 0 & 0.0385 & 0.0402 & 0 & 0 & 0.0263 & 0.0279 & 0 \\
0 & 0 & 0 & 0 & 0 & 0 & 0 & 0 & 0 & 0.0823 & 0.0845 & 0 & 0 & 0.0563 & 0.0584 & 0 & 0 & 0.0385 & 0.0402 & 0 \\
0 & 0 & 0 & 0 & 0 & 0 & 0 & 0 & 0 & 0 & 0 & 0 & 0 & 0.0823 & 0.0845 & 0 & 0 & 0.0563 & 0.0583 & 0 \\
0 & 0 & 0 & 0 & 0 & 0 & 0 & 0 & 0 & 0 & 0 & 0 & 0 & 0 & 0 & 0 & 0 & 0.0823 & 0.0845 & 0 \\
0 & 0 & 0 & 0 & 0 & 0 & 0 & 0 & 0 & 0 & 0 & 0 & 0 & 0 & 0 & 0 & 0 & 0 & 0 & 0
\end{array}\right]^{\mathrm{T}} \\
H_{C 2} & =\left[\begin{array}{llllllllllllllllllll}
0 & 0 & 0 & 0 & 0.00224 & 0 & 0.0845 & 0 & 0.00197 & 0 & 0.0583 & 0 & 0.00174 & 0 & 0.0402 & 0 & 0.00154 & 0 & 0.0279 & 0 \\
0 & 0 & 0 & 0 & 0 & 0 & 0 & 0 & 0.00224 & 0 & 0.0845 & 0 & 0.00197 & 0 & 0.0583 & 0 & 0.00174 & 0 & 0.0402 & 0 \\
0 & 0 & 0 & 0 & 0 & 0 & 0 & 0 & 0 & 0 & 0 & 0 & 0.00224 & 0 & 0.0845 & 0 & 0.00197 & 0 & 0.0583 & 0 \\
0 & 0 & 0 & 0 & 0 & 0 & 0 & 0 & 0 & 0 & 0 & 0 & 0 & 0 & 0 & 0 & 0.00224 & 0 & 0.0845 & 0 \\
0 & 0 & 0 & 0 & 0 & 0 & 0 & 0 & 0 & 0 & 0 & 0 & 0 & 0 & 0 & 0 & 0 & 0 & 0 & 0
\end{array}\right]^{\mathrm{T}}
\end{aligned}
$$$$
H_{C 3}=\left[\begin{array}{cccccccccccccccccccccccccc}
0 & 0 & 0 & 0 & 0.00224 & 0.0823 & 0 & 0 & 0.00197 & 0.0563 & 0 & 0 & 0.00174 & 0.0385 & 0 & 0 & 0.00154 & 0.0263 & 0 & 0 \\
0 & 0 & 0 & 0 & 0 & 0 & 0 & 0 & 0.00224 & 0.0823 & 0 & 0 & 0.00197 & 0.0563 & 0 & 0 & 0.00174 & 0.0385 & 0 & 0 \\
0 & 0 & 0 & 0 & 0 & 0 & 0 & 0 & 0 & 0 & 0 & 0 & 0.00224 & 0.0823 & 0 & 0 & 0.00197 & 0.0563 & 0 & 0 \\
0 & 0 & 0 & 0 & 0 & 0 & 0 & 0 & 0 & 0 & 0 & 0 & 0 & 0 & 0 & 0 & 0.00224 & 0.0823 & 0 & 0 \\
0 & 0 & 0 & 0 & 0 & 0 & 0 & 0 & 0 & 0 & 0 & 0 & 0 & 0 & 0 & 0 & 0 & 0 & 0 & 0
\end{array}\right]^{\mathrm{T}}
$$$$
H_{C 4}=\left[\begin{array}{ccccccccccccccccccccc}
0 & 0 & 0 & 0 & 0.00224 & 0.0823 & 0.0845 & 0 & 0.00197 & 0.0563 & 0.0583 & 0 & 0.00174 & 0.0385 & 0.0402 & 0 & 0.00154 & 0.0263 & 0.0279 & 0 \\
0 & 0 & 0 & 0 & 0 & 0 & 0 & 0 & 0.00224 & 0.0823 & 0.0845 & 0 & 0.00197 & 0.0563 & 0.0583 & 0 & 0.00174 & 0.0385 & 0.0402 & 0 \\
0 & 0 & 0 & 0 & 0 & 0 & 0 & 0 & 0 & 0 & 0 & 0 & 0.00224 & 0.0823 & 0.0845 & 0 & 0.00197 & 0.0563 & 0.0583 & 0 \\
0 & 0 & 0 & 0 & 0 & 0 & 0 & 0 & 0 & 0 & 0 & 0 & 0 & 0 & 0 & 0 & 0.00224 & 0.0823 & 0.0845 & 0 \\
0 & 0 & 0 & 0 & 0 & 0 & 0 & 0 & 0 & 0 & 0 & 0 & 0 & 0 & 0 & 0 & 0 & 0 & 0 & 0
\end{array}\right]^{\mathrm{T}}
$$

\section{CONCLUSION}

An approach based on the fully decoupled parity equation for fault diagnosis in systems with unknown nonlinearity is presented in this paper. Conditions for the residuals generated from the fully decoupled parity equation to be sensitive only to specific faults, but decoupled from the system state, the unknown nonlinearity, and other faults are derived. By assuming the actuator and sensor faults to be approximated by a linear fault model, these faults can be estimated from the residuals by the recursive least-squares method. It is shown that the proposed method has successfully detected and identified multiple actuator or sensor faults in a simulated dc motor.

\section{REFERENCES}

[1] J. Gertler, Fault Detection and Diagnosis in Engineering Systems. New York: Marcel Dekker, 1999.

[2] J. Chen and R. J. Patton, Robust Model-Based Fault Diagnosis for Dynamic Systems. Norwell, MA: Kluwer, 1999.

[3] J. Korbicz, J. M. Koscielny, Z. Kowlaczuk, and W. Cholewa, Fault Diagnosis. Berlin, Germany: Springer-Verlag, 2004.

[4] J. E. Pottor and M. C. Suman, "Thresholdless redundancy management with arrays of skewed instruments," NATO AGARDOGRAPH on Integrity Electron. Flight Control Syst., no. 224, pp. 15-11-15-25, 1977.

[5] J. C. Deckert, M. N. Desai, J. J. Deyst, and A. S. Willsky, "DFBW sensor failure identification using analytic redundancy," IEEE Trans. Autom. Control, vol. AC-22, no. 5, pp. 795-809, Oct. 1977.

[6] K. C. Daly, E. Gai, and J. V. Harrison, "Generalized likelihood test for FDI in redundant sensor configurations," J. Guidance Control, vol. 2, no. 1, pp. 9-17, Feb. 1979.

[7] M. Desai and A. Ray, "A fault detection and isolation methology," in Proc. 20th Conf. Decision Control, 1981, pp. 1363-1369.

[8] E. Y. Chow and A. S. Willsky, "Analytical redundancy and the design of robust failure detection systems," IEEE Trans. Autom. Control, vol. AC-29, no. 7, pp. 603-614, 1984.

[9] X. C. Lou, A. S. Willsky, and G. C. Verghese, "Optimally robust redundancy relations for failure detection in uncertain systems," Automatica, vol. 22, no. 3, pp. 333-344, May 1986.
[10] H. Y. Zhang and R. J. Patton, "Optimal design of robust analytical redundancy for uncertain systems," in Proc. IEEE Region 10 Conf. Comput., Commun., Control and Power Eng., Beijing, China, 1993, pp. 218-221.

[11] J. Hong and H. Y. Zhang, "Optimal parity vector sensitive to designated sensor fault," IEEE Trans. Aerosp. Electron. Syst., vol. 35, no. 4, pp. 1122-1128, Oct. 1999.

[12] _ , "FDI of slow-grown faults of dynamic systems," (in Chinese) Aerosp. Control, vol. 18, no. 72, pp. 65-70, Apr. 2000.

[13] J. Y. Tu and J. L. Stein, "Model error compensation for observer design," Int. J. Control, vol. 69, no. 2, pp. 329-345, Jan. 1998.

[14] M. Hou and P. C. Muller, "Design of observers for linear systems with unknown inputs," IEEE Trans. Autom. Control, vol. 37, no. 6, pp. 137-141, Jun. 1992.

[15] M. Darouach, M. Zasadzinski, and S. J. Xu, "Full-order observer for linear systems with unknown inputs," IEEE Trans. Autom. Control, vol. 39, no. 3, pp. 606-609, Mar. 1994.

[16] M. Hou and P. C. Muller, "Fault detection and isolation observers," Int. J. Control, vol. 60, no. 5, pp. 827-846, 1994.

[17] S. K. Chang and P. L. Hsu, "A novel design for the unknown input fault-detection observer," Control Theory Adv. Technol., vol. 10, no. 4, pp. 1029-1051, 1995.

[18] J. Lunze and J. Schröder, "Sensor and actuator fault diagnosis of systems with discrete inputs and outputs," IEEE Trans. Syst., Man, Cybern. B, Cybern., vol. 34, no. 2, pp. 1096-1107, Apr. 2004.

[19] S. J. Qin and W. Li, "Detection, identification, and reconstruction of faulty sensors with maximized sensitivity," AIChE J., vol. 45, no. 9, pp. 1963-1976, Sep. 1999.

[20] X. Wen, H. Y. Zhang, and L. Zhou, Fault Diagnosis and Fault Tolerant Control for Control System. Beijing, China: Mach. Ind. Press, 1998. (in Chinese).

[21] G. Schram, S. Gopisetty, and R. F. Stengel, A Fuzzy Logic-Parity Space Approach to Actuator Failure Detection and Identification. Washington, DC: AIAA, 1998, pp. 98-1014.

[22] C. S. Leung, K. W. Wong, P. F. Sum, and L. W. Chan, "On-line training and pruning for recursive least square algorithms," Electron. Lett., vol. 32, no. 23, pp. 2152-2153, Nov. 7, 1996.

[23] H. Song and H. Y. Zhang, "An approach to sensor fault diagnosis based on fully-decoupled parity equation and parameter estimate," in Proc. 4th World Congr. Intell. Control and Autom., Shanghai, China, Jun. 2002, pp. 2750-2754.

[24] O. Moseler and R. Isermann, "Application of model-based fault detection to a brushless dc motor," IEEE Trans. Ind. Electron., vol. 47, no. 5, pp. 1015-1020, Oct. 2000. 
[25] X.-Q. Liu, H. Y. Zhang, J. Liu, and J. Yang, "Fault detection and diagnosis of permanent-magnet DC motor based on parameter estimation and neural network," IEEE Trans. Ind. Electron., vol. 47, no. 5, pp. 1021-1030, Oct. 2000.

[26] K. Watanwbe, M. Sasak, and D. M. Himmelblau, "Determination of optimal measuring sites for fault detection of non-linear systems," Int. J. Syst. Sci., vol. 16, no. 11, pp. 1345-1363, 1985.

[27] Y. Wang, "On-line fault diagnosis of nonlinear dynamical systems using recurrent neural network," Ph.D. dissertation, Univ. Hong Kong, Hong Kong, 2000.

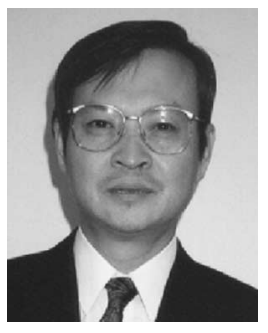

C. W. Chan received the Higher Diploma degree in mechanical engineering from Hong Kong Polytechnic University, Hong Kong, and the M.Sc. and the Ph.D. degrees from the Control Systems Centre, University of Manchester Institute of Science and Technology, Manchester, U.K.

He was with Weir Pumps PLC, the National Engineering Laboratory, and Unilever Research Port Sunlight Laboratory before joining The University of Hong Kong, Hong Kong. His current research interests include fault detection, isolation and identification for nonlinear systems, compensation for nonlinear actuators, fuzzy logic control, and neural-networks-based modeling and control.

Dr. Chan is a Regional Editor of the International Journal of Systems Science and an Associate Editor of the Transactions of the Hong Kong Institution of Engineers. He is a member of the Hong Kong Institution of Engineers, the Institution of Mechanical Engineers, the Institution of Electrical Engineers, and an Associate Fellow of the Institute of Mathematics and Its Applications.

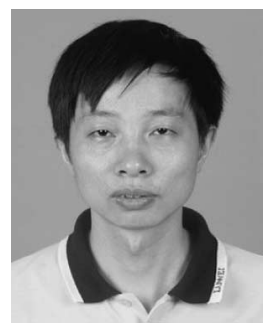

Song Hua received the degree and the Ph.D. degree, for his work on fault diagnosis, from Beihang University, Beijing, China, in 1992 and 2002, respectively.

$\mathrm{He}$ is currently a Lecturer in the School of Automation Science and Electrical Engineering, Beihang University. His main interests are in the fields of fault diagnosis and reliability analysis for nonlinear systems.

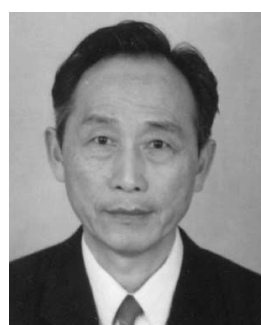

Zhang Hong-Yue (SM'91) received the degree from Beihang University, Beijing, China.

From 1979 to 1981, he was a Visiting Scientist at Princeton University, Princeton, NJ, and Houston University, Houston, TX. He is currently a Professor in the School of Automation Science and Electrical Engineering, Beihang University. He has published about 200 papers and five books in his fields of research interest. His research interests include faulttolerant control and navigation, fault detection and diagnosis, reliability of control systems, system identification and state estimation, and neural networks and their applications. 